\title{
Modular Invariance, Self-Duality and The Phase Transition Between Quantum Hall Plateaus
}

\author{
Eduardo Fradkin* and Steven Kivelson ${ }^{\dagger}$ \\ Department of Physics, University of Illinois at Urbana-Champaign* , 1110 W.Green St, Urbana, \\ IL 61801-3080 and Department of Physics, University of California Los Angeles ${ }^{\dagger}$, CA 90024
}

\begin{abstract}
We investigate the problem of the superuniversality of the phase transition between different quantum Hall plateaus. We construct a set of models which give a qualitative description of this transition in a pure system of interacting charged particles. One of the models is manifestly invariant under both Duality and Periodic shifts of the statistical angle and, hence, it has a full Modular Invariance. We derive the transformation laws for the correlation functions under the modular group and use them to derive symmetry constraints for the conductances. These allow us to calculate exactly the conductivities at the modular fixed points. We show that, at least at the modular fixed points, the system is critical. Away from the fixed points, the behavior of the model is determined by extra symmetries such as Time Reversal. We speculate that if the natural connection between spin and statistics holds, the model may exhibit an effective analyticity at low energies. In this case, the conductance is completely determined by its behavior under modular transformations.
\end{abstract}

Typeset using REVTEX 


\section{INTRODUCTION}

The problem of the nature of the phase transitions between the plateaus of the Quantum Hall States is one of the most challenging questions in the physics of strongly correleted Fermi systems. These phase transitions separate states whose most important physical feature is their inherent topological nature. Thus, it has been shown that the fluid ground states of Quantum Hall systems have a form of topological order, instead of conventional long range order [1]. Furthermore [2] [4], the low-lying excitations are fractionally charged quasiparticles with fractional statistics [5]. A direct consequence of the topological order is the universal properties of the edge states [6]. In addition to the values of the Hall conductance, the plateaus are uniquely defined by the quantum numbers of their excitations. Hence, the effective theories which describe the plateau behaviors must be topological field theories. It has been known for some time that Chern-Simons gauge theories give an accurate description of the plateaus [7].

It is then natural to ask if the behavior of the system at the phase transtition between a pair of plateaus depends on the properties of the plateaus. A theory of the transition between Hall plateaus was proposed recently by Kivelson, Lee and Zhang (KLZ) [8]. This theory explains the rich phase diagram seen experimentally. KLZ used a bosonic description of the FQHE and, in this picture, the transition between plateaus becomes strongly reminiscent to the superfluid-insulator phase transition in a disordered system. The transition beteween plateaus appears to be generally a first order transition in the pure system but it is smoothed to a second order transtion by effects of disorder. In this picture, the stable FQHE plateaus

are the phases in which the Hall conductivity $\sigma_{x y}=\nu \frac{e^{2}}{h}$ has precisely defined values. Here $\nu$ is a fraction whose denominator is always odd. In this state the longitudinal conductivity vanishes exactly, $\sigma_{x x}=0$. Since in such theories the Hall conductivity is the coefficient of the effective Chern-Simons term for the external electromagnetic field, the transition between plateaus becomes a transition between states with effective actions for the electromagnetic field with different Chern-Simons coefficients. At the transition $\sigma_{x x} \neq 0$ (and perhaps 
universal) and the denominator of $\sigma_{x y}$ is even. In the language of the renormalization group, the plateaus are represented by stable fixed points described by effective actions which have a pure Chern-Simons form [9,10]. From this viewpoint it is natural to ask if the critical properties at the transtion between plateaus depends on the precise values of the Chern-Simons coefficients of the fixed points or if there is some sort of superuniversality at work. Kivelson, Lee and Zhang [8] have argued that the localization length exponent at the transition between plateaus in a disordered quantum Hall system should be the same for all these phase transitions, while the longitudinal conductivity at the transition may be universal although different for different transitions. Hence, KLZ have argued that these transtions are superuniversal. Furthermore, Lütken and Ross [11,12] have conjectured that the theories for the FQHE posses an effective modular invariance which seemingly implies such a superuniversality in the sense that all the critical points have the same critical exponents and the critical conductances are related by modular transformations. Recent Monte Carlo simulations on a related system by Manousakis [13] seem to support the notion of superuniversality.

Recent experimental work by Shahar et. al. [14] on the transition between quantum Hall and insulating states have revealed an effective self-duality relating the properties of the system on either side of the phase transition. This self-duality is observed in a suprisingly broad neighborhood of the critical point. This observation gives strong motivation to study systems with particle-vortex duality. Of course such a symmetry can emerge near the phase transition even if the microscopic theory is not self-dual.

Chern-Simons gauge field theories thus provide a natural description of the plateaus of the fractional quantum Hall effect (FQHE) as well as of other problems in condensed matter physics and field theories in two space dimensions. Chern-Simons theories appear in the effective theories for the low energy physics [7,15,16] (in the Landau-Ginzburg sense). Their presence makes manifest the breaking of time reversal invariance in the underlying system. Chern-Simons theories are also the natural framework for statistical transmutation [17 21]. In particular, in the FQHE Chern-Simon theories appear in the form of exact mappings 
between theories in which the particles which bear the same electric charge but, in general, have different statistics [7,22,23]. This latter approach has led to two mutually complementary descriptions of the FQHE and its hierarchies. One is based on a bosonic picture within which a superfluid analogy plays a central role. The other uses a fermionic picture with an integer Hall effect as the reference analogy [24].

Motivated by these considerations, in this paper we consider the critical properties of phase transitions in theories of matter coupled to a Chern-Simons gauge field. Our main goal is to investigate if the critical properties of such systems are altered in any relevant way by the coupling of the order parameter field to a fluctuating Chern-Simons gauge field. In contrast with the generally accepted assumption that the transition between plateaus is necessarily a first order transition in a pure system, we construct a model in which the transition is apparently second order, even in the abscence of disorder. This model can serve as the prototype of the phase transition between plateaus. The strategy that we follow in this work is to construct physically reasonable models which manifestly have these symmetries and to use them to describe the plateaus and the phase transitions. The models should also yield the correct electromagnetic response functions at its stable fixed points which will then be identified with the plateaus.

More specifically, we consider systems of interacting particles with fractional statistics parametrized by an angle $\delta$. In Chern-Simons language, this corresponds to a coupling constant $\theta=1 / 2 \delta$. In order to address the issue of superuniversality it is necessary to define carefully the symmetries exhibited by these systems, even at the microscopic level. The problem discussed here is the same as to ask if the universality classes of a phase transition depends on the fractional statistics [25]. The notion of fractional statistics is clearly well defined for systems with a finite number of particles (first quantization). In this case, it is straightforward to check that all theories of this type are invariant under a shift of the statistical angle $\delta \rightarrow \delta+2 \pi k$ (where $k$ is an integer) or, equivalently, $\frac{1}{\theta} \rightarrow \frac{1}{\theta}+4 \pi k$. This is the invariance of the theory of the FQHE under the attachment of an even number $2 k$ of flux quanta to each particle. We will call this symmetry Periodicity and denote it by $P$ 
(not to be confused with parity!). Also, in systems in which particle-hole symmetry is exact, the theory is invariant under a change in the sign of the Chern-Simons coupling constant, $\theta \rightarrow-\theta$. For obvious reasons, we will call this symmetry Charge Conjugation and denote it by $C$. Finally, we will show that there is another symmetry at work in these systems, Duality, which we will call $S$. However, as in other situations in statistical physics, Duality is not a symmetry but rather it maps different theories among each other. We show however that it is possible to construct exactly self-dual theories which will serve as fixed points.

In this paper we construct two classes of models, which we call Model I and II respectively. Both models exhibit quantum critical behavior at zero temperature, In Model I, at least, this behavior is associated with zero temperature phase transitions. Model II appears to be critical at zero temperature for all physically meaningful values of the coupling constants. Both types of model are explicitly Periodic in the statistical angle and, in addition, Model II is explicitly self-dual.

Model I describes a system of charged particles with (in general) fractional statistics and short range interactions. These models are not self-dual in the sense that the form of the interaction is not in general preserved by the Duality transformation. In fact, there are cases in which a theory of particles with short range interactions is dual to a theory of vortices (the particles of the dual theory) which have long range interactions. This is in fact the familiar case of a superfluid-insulator transition. In general, the stable phases of the systems described by Model I have the qualitative properies of Quantum Hall plateaus and it is qualitatively similar to the Landau-Ginzburg theory of the FQHE. This model gives the correct plateau behavior. However, in general, it is not self-dual and its properties at the phase transtion betweeen plateaus are not understood. Recently, Pryadko and Zhang [26] and Balachandran, Chandar and Sathialpan [27 have considered a model closely related to Model I and discussed its properties under duality transformations. Our conclusions on the physics of these model agrees with theirs.

The physics of the transitions between Quantum Hall plateaus strongly suggests that, at least very close to the transition, the system should be effectively self-dual. This motivates 
the construction of a model, Model II, which is explicitly self-dual. However, Model II will turn out to have interactions which are long ranged and retarded. Physically, Model II describes the behavior of a system whose excitations are charged particles with fractional statistics, restricted to move on a plane. The long-range retarded interactions are due to the quantum fluactuations of the electromagnetic field in the full $3+1$-dimensional spacetime [28]. Naturally, when the coupling constant of the long-range interactions becomes very small, the short range interactions (of the sort described by Model I) become dominant.

We will show that Model II exhibits quantum critical behavior of a sort that is readily analyzed. We conjecture that this behavior is characteristic of the behavior of a broader class of systems at their zero temperature critical points, including Model I. Model II is manifestly periodic under shifts of the statistical angle $\delta$. We find it convenient to define a parameter $f=\delta / 2 \pi$. The interactions between these particles is represented by a longranged kernel which at long wavelenths we choose to be of the form $2 \pi g / \sqrt{k^{2}}$, where $k_{\mu}$ is the three-momentum ( $i$.e., momentum and frequency). The parameter $g$ is be the coupling constant and, for stability, it must be positive (repulsive). This model is both self-dual and periodic. We show that the partition function of Model II is invariant under the Duality transformations

$$
g \rightarrow \frac{g}{g^{2}+f^{2}} \quad f \rightarrow-\frac{f}{g^{2}+f^{2}}
$$

as well as under Periodicity

$$
f \rightarrow f+1
$$

These two transformations do not commute with each other. Instead, they generate an infinite discrete group known as the Modular Group or $S L(2, Z)$. Thus these systems are invariant under that action of $S L(2, Z)$. In fact, in terms of the complex variable $z=f+i g$ (which has $\operatorname{Im} z=g>0$ ), the group of Modular transformations becomes the group of fractional linear transformations of the complex plane with integer coefficients

$$
z \rightarrow \frac{a z+b}{c z+d}
$$


where $a, b, c$ and $d$ are integers such that $a d-b c=1$.

Since Model II is manifestly invariant under both Periodicity and Duality, its partition function is Modular Invariant. The Modular Group $S L(2, Z)$ has a rich structure. In particular it has an infinite set of fixed points, namely points of the upper half plane which are invariant under at least one, non-trivial modular transformation. We will refer to these points as the modular fixed points. Lütken and Ross [12 have used this structure as the basis for a conjecture concerning the behavior of possible renormalization group flows in QHE systems. However, in the work of Lütken and Ross, $S L(2, Z)$ acts directly on the complex conductance $\sigma_{x y}+i \sigma_{x x}$. Consequently, the fixed points of $S L(2, Z)$ in reference [12 are the allowed values of the complex conductances. In contrast, in Model II the modular fixed points are points of special symmetry. In analogy with the Kramers-Wannier argument for the two-dimensional Ising Model [29], we can expect the phase transitions to occur at the modular fixed points. Nevertheless, we will show below that Modular Invariance is enough to determine the conductance of Model II, at least at the modular fixed points. However, we will also find that there are other values of $f$ and $g$ where the system is critical.

Having an explicit construction of a model which is modular invariant has allowed us to investigate the consequences of modular invariance on physical observables. In particular, we show that the current correlation functions of these models transform (almost) like a modular form. More precisely, we show that from the current-current correlation function of this system it is possible to construct an amplitude, which we call $D(z)$, which determines the complex conductance. By construction, $D(z)$ is Periodic under $z \rightarrow z+1$ and, under Duality, it transforms like $D(-1 / z)=z^{2} D(z)+z$. Combining both transformations we find that, under a general modular transformation, $D(z)$ transforms like

$$
D\left(\frac{a z+b}{c z+d}\right)=(c z+d)^{2} D(z)+c(c z+d)
$$

This functional relation is a direct consequence of the modular invariance of the partition function and of the fact that this invarience is broken by an external electromagnetic field in a well defined manner. In fact, although we derived this functional relation in the context 
of Model II, we expect it to be generally valid for any modular invariant system. What is very interesting is the fact that this functional relation is sufficient to determine the values of the conductances for all the fixed points of the modular group $S L(2, Z)$. Below, we derive a formula which yields the allowed values of the conductance at the modular fixed points. Furthermore, by making additional assumtions on the possible analytic properties of the correlation functions compatible with the symmetries of the system, we can also determine their behavior away from the modular fixed points. In particular, modular invariance requires that, quite generally, $\sigma_{x x}$ is finite, at least at the modular fixed points.

Interacting charged particles without impurities are generally expected to be either insulators or perfect metals (or even superconductors). Correspondingly $\sigma_{x x}=0$ for an insulator while $\sigma_{x x}=\infty$ for a perfect metal (or superconductor). In order to find $\sigma_{x x} \neq 0$ (at zero temperature) it is usually assumed that the system must be disordered (or break galilean invariance in some way). However, there is an alternative scenario which yields a finite $\sigma_{x x}$ in a non-disordered system: a Quantum Critical Point. At a Quantum Critical Point, the spectral functions of all the correlation functions are changed drastically by the effects of fluctuations on all length scales. In particular, the pole structure associated with the existence of well defined elementary excitations is wiped out and replaced by branch cuts. Hence, the spectrum becomes a broad incoherent continuum. A direct consequence of this behavior is that $\sigma_{x x}$ can now be finite. Thus, we will interpret having a finite $\sigma_{x x}$ in a (pure) system of charged interacting particled as a signature of a Quantum Critical Point.

Some time ago C. J. Callan and D. E. Freed found in their work on the Dissipative Hofstadter Model [30] an algebraic structure very similar to the one we use in this paper. It is clear that Model II is quite analogous to the Dissipative Hofstadter Model. In particular, our coupling constant $g$ plays the role of the dissipation in the Dissipative Hofstadter Model. Similarly, the Modular Group also played a central role in the work of Shapere and Wilczek [31] and of Rey and Zee [32] on self-dual $Z_{N}$ models in $2+1$ dimensions.

The paper is organized as follows. In Section $\Pi$ we introduce a class of models which are manifestly invariant under periodic shifts of the statistical angle. In Section III we 
construct the Duality transformation. In particular, we discuss the way the presence of external electromagnetic perurbations alter the way these systems transform under Duality. In Section $\mathbb{\| \nabla}$ we discuss the electromagnetic response functions and their properties under Duality. In Section $\mathrm{V}$ we introduce Model I and Model II and discuss the nature of their phase diagrams. This section contains the most important results of this paper, including the determination of the complex conductance at the modular fixed points. In Section VI we discuss the role of periodicity in quantum field theories of matter coupled to Chern-Simons. Section VII is devoted to the conclusions.

\section{MODELS}

For all the reasons invoked above we will adopt a lattice formulation in which all symmetries are manifest. We will work in discretized $2+1$ dimensional Euclidean space-time. We consider a three-dimensional cubic lattice with sites $\{\vec{r}\}$ and links $\{\vec{r}, \mu\}(\mu=1,2,3$ where 1 and 2 are the space directions and 3 is (imaginary) time). We represent the worldlines of the charged particles by means of an integer valued conserved vector field $\ell_{\mu}(\vec{r})$ defined on the links of this lattice. Conservation means, naturally, that $\Delta_{\mu} \ell_{\mu}(\vec{r})=0$ at every site $\vec{r}$, where $\Delta_{\mu} \ell_{\mu}$ is the lattice divergence. The current loops can be either the worldlines of first quantized particles relative to the empty state or the worldlines of excitations relative to a reference ground state. Here we envision several physically distinct situations. One system we want to describe cosnsits of non-relativistic charged particles with some statistics and at finite density. In this case, the worldlines do not end and only close around the time dimension. Another set of cases of interest are relativistic systems (at zero density). In these cases, closed loops are allowed and represent particle-antiparticle pairs. Relativistic field theories are Charge Conjugation invariant. Non-relativistic systems with or without Charge Conjugation symmetry fit this description as well if one now thinks of the loops as representing quasiparticle-quasihole pairs. When we compute the electromagnetic response of these systems, we will have to bear in mind that, in general, there is a non-trivial contribution 
from the reference vacuum which must be added to the contributions of the excitations.

Thus, we are interested in the behavior of theories which have both closed loops and with loops that close around the time dimension. The loops can be described in terms of a set of bare particles which can be taken to be bosons but their actual statistics is determined by a topological term in the action. Typically, this is accomplished by coupling matter to a Chern-Simons gauge field [7]. Since we want to make all symmetries explicit we choose to define the theory on a lattice. Chern-Simons theories have been defined on lattices but they are rather cumbersome to work with. Thus, we use an equivalent construction in which we directly attach fluxes to particles and, in this way achieve statistical transmutation [17. The simplest way to incorporate statistical transmutation in a discretized picture is to modify the weight of the path integral by a factor which is a pure phase equal to the statistical angle $\delta$ times the linking number $\nu_{L}$ of the worldlines of the particles. Theories with the property of charged excitations always being bound to magnetic charge (or flux) are said to exhibit oblique confinement [33]. The Landau-Ginzburg theories of the FQHE [7] are simplest (and best understood) example of oblique confinement [34].

In order to keep track of particles and fluxes, and to avoid ambiguities, we define the worldlines of the fluxes in such a way that they strictly follow the matter worldlines but exist on neighboring sites of the dual lattice, which we denote by $\{\vec{R}\}$. Thus, the configuration of fluxes on the links of the dual lattice is the same as the configuration of matter on the links of the direct lattice, $i$. e. $\ell_{\mu}(\vec{r})=\ell_{\mu}(\vec{R})$ where $\vec{R} \equiv \vec{r}+\left(\frac{1}{2}, \frac{1}{2}, \frac{1}{2}\right)$. Thus, the vector field $\ell_{\mu}$ represents both the world lines of the particles and the worldlines of the fluxes which, therefore, do not have an independent existence. As a consequence, charges cannot detect the constituent fluxes of other particles without detecting their charge.

Notice that, if the fluxes are attached to the worldlines of the particles by a rigid translation, then the system is defined in such a way that the particle cannot feel its own flux. In this case there will be no phase factor associated with the writhing number (or selfwinding) of the worldline. Hence, there is no fractional spin associated with the configurations but there will be fractional statistics. However, one can imagine other definitions of the system 
at short distances. For instance, it is possible to construct the flux-charge composite in such a way that the flux rotates around the worldline as the latter winds around itself. In this case the flux-charge composite becomes the standard rubber band construction associated with the framing of knots. It is well known from the work of Polyakov [20] and Witten [19] that this construction leads to the conventional spin-statistics connection. With our explicit construction there is no fractional spin, so the theory has the invariance $\theta \rightarrow-\theta$ discussed in the Introduction. We will show below that the presence of this symmetry imposes severe constraints on the behavior of the correlation functions. Conversely, if a fractional spin is associated with the flux-charge composite, this symmetry will be broken by an amount determined by the fractional spin. (We return to this point below.)

The action $S\left[\ell_{\mu}\right]$ for the field $\ell_{\mu}(\vec{r})$ will be chosen to be of the form

$$
\begin{aligned}
S\left[\ell_{\mu}\right]= & \frac{1}{2} \sum_{\vec{r}, \vec{r}^{\prime}} \ell_{\mu}(\vec{r}) G_{\mu \nu}\left(\vec{r}-\vec{r}^{\prime}\right) \ell_{\nu}\left(\vec{r}^{\prime}\right)+\frac{i}{2} \sum_{\vec{r}, \vec{R}} \ell_{\mu}(\vec{r}) K_{\mu \nu}(\vec{r}, \vec{R}) \ell_{\nu}(\vec{R}) \\
& +i \sum_{\vec{r}, \vec{r}^{\prime}} e\left(\vec{r}-\vec{r}^{\prime}\right) \ell_{\mu}(\vec{r}) A_{\mu}\left(\vec{r}^{\prime}\right)+\sum_{\vec{R}, \vec{R}^{\prime}} h\left(\vec{R}-\vec{R}^{\prime}\right) \ell_{\mu}(\vec{R}) B_{\mu}\left(\vec{R}^{\prime}\right) \\
& +\frac{1}{2} \sum_{\vec{r}, \vec{r}^{\prime}} A_{\mu}(\vec{r}) \Pi_{\mu \nu}^{0}\left(\vec{r}, \vec{r}^{\prime}\right) A_{\nu}\left(\vec{r}^{\prime}\right)
\end{aligned}
$$

The partition function is

$$
\mathcal{Z}=\sum_{\left\{\ell_{\mu}\right\}} \prod_{\vec{r}} \delta\left[\Delta_{\mu} \ell_{\mu}(\vec{r})\right] e^{-S\left[\ell_{\mu}\right]}
$$

where $\{\vec{r}\}$ are the sites of the direct lattice while $\{\vec{R}\}$ are the sites of the dual lattice, i. e. $\vec{R}=\vec{r}+\left(\frac{1}{2}, \frac{1}{2}, \frac{1}{2}\right)$. Here $A_{\mu}(\vec{r})$ is an external electromagnetic field and $B_{\mu}(\vec{R})$ is the corresponding field strength. $A_{\mu}(\vec{r})$ couples to matter through two coupling constants, an electric charge $e$ and a magnetic charge $h$.

The physical interpretation of each term of the action of Eq. (2.1) is as follows:

1. The matter interactions are represnted by $G_{\mu \nu}\left(\vec{r}-\vec{r}^{\prime}\right)$. This is the parity even part of the action and it describes the repulsive forces among the particles. For instance, the short range part of $G_{\mu \nu}\left(\vec{r}-\vec{r}^{\prime}\right)$ essentially suppresses configurations with $\left|\ell_{\mu}\right|>1$ which means that it penalizes the occupancy of one link by more than one particle. 
Physically, $G_{\mu \nu}\left(\vec{r}-\vec{r}^{\prime}\right)$ is the propagator of some field that mediates the interactions between the particles. The explicit form of the kernel $G_{\mu \nu}\left(\vec{r}-\vec{r}^{\prime}\right)$ will be given below.

2. The statistical interactions induced by Chern-Simons gauge fields are represented by the tensor $K_{\mu \nu}\left(\vec{r}, \vec{R}^{\prime}\right)$ and it is parity odd. The precise form on a lattice of this tensor is rather complicated (see for instance reference [35]). For our purposes, it will be sufficient to require that it assigns the correct phase factors depending on the braiding of the worldlines. The continuum Chern-Simons action (for a gauge field $\mathcal{A}_{\mu}$ ) is $S_{\mathrm{CS}}\left(\mathcal{A}_{\mu}\right)=\frac{\theta}{2} \epsilon_{\mu \nu \lambda} \mathcal{A}_{\mu} \partial_{\nu} \mathcal{A}_{\lambda}$, where $\theta$ is the Chern-Simons coupling constant. Then 19,20 the statistical angle $\delta$ and the coupling constant $\theta$ are related by $\delta=1 /(2 \theta)$. The weight for a configuration is $\exp \left(i \delta \nu_{L}\right)$, where $\nu_{L}$ is the linking number of the configuration [20,21]. Using a magnetostatic analogy, it is possible to find [20] a simple formula which relates the linking number $\nu_{L}$ to a set of currents $J_{\mu}$ which describe the worldlines. Explicitly one finds [20]

$$
\nu_{L}=\int d^{3} x \int d^{3} y J_{\mu}(x) \epsilon_{\mu \nu \lambda} G_{0}(x-y) \partial_{\lambda} J_{\nu}(y),
$$

where $G_{0}(x-y)$ is the three-dimensional green's function,

$$
-\nabla^{2} G_{0}(x-y)=\delta(x-y) .
$$

Thus we define $K_{\mu \nu}$ so that, to each configuration of matter, the weight of the pathintegral has a phase factor with argument equal to the linking number of the configuration up to a coupling constant which we identify with the statistical angle $\delta$. In other words, the term involving the tensor $K_{\mu \nu}$ represents fractional statistics. By construction, it is automatically invariant under periodic shifts of the statistical angle. The explicit form of $K_{\mu \nu}$ depends on details of the discretization procedure. We will use an explicit form which works provided that the arguments of $K_{\mu \nu}$ are defined to be one on the direct lattice and the other on the dual lattice. In continuum description, a point-splitting regularization is needed to define the linking number. The lattice regularization takes care of this problems automatically. 
3. The terms in the second line of the action of eq. 2.1 represent the coupling to an external vector field $A_{\mu}(\vec{r})$. The space components, 1 and 2 , of this vector field can be used to implement an external magnetic field. The time component (3) is a scalar potential and a constant piece represents a chemical potential (up to an analytic continuation) since it counts the net charge flowing forwards in time at a given time slice. In general, $A_{\mu}(\vec{r})$ will be an external electromagnetic field that we will use to probe the system. The electric coupling $e(\vec{r})$ is the conventional lattice version of the usual current coupling which we will assume to be mildlyly non-local. The coupling of the vortex current to the field strength $B_{\mu}, h(\vec{R})$, is natural as it represents the coupling between the field and and the circulating current associated with the vorticity (in the sense of Ampère's Law). In any event, this term gets generated automatically under duality even if it was not present in the starting hamiltonian. Similarly, we have allowed these couplings to be non-local since they will generally become non-local under duality transformations. In any case at the fixed points that we will find the non locality of the electric coupling is irrelevant.

4. The last term of the action represents the electromagnetic response of background degrees of freedom which do not participate of the dynamics but which affect the electrodynamics of the system (e.g., the conductances, etc.)

Since we are interested in configurations of loops (worldlines) which are large and well separted from each other, we do not need to specify the details of the construction of the kernels $G_{\mu \nu}$ and $K_{\mu \nu}$ at short distances. The precise form of these kernels at distance scales of the order of the lattice constant will not affect the universal, long distance, properties. Thus, in practice, we will only specify their behavior at long distances or small momenta. The only assumption we will make here is that the models of interest can be defined on a lattice, in such a way that all the symmetries are preserved. 


\section{DUALITY}

The strategy of this paper is thus to classify the stable phases and phase transitions of theories with the action of eq. (2.1) (and a number of simple generalizations). The most direct way to do this is to use duality transformations [29. Duality has been used with great success in the study of theories with a structure similar to the one we discussed here 36 38. Of particular importance to the problems discussed here, is the work of Cardy and Rabinovici [39] on theories of two-dimensional statistical mechanics with oblique confinement. Cardy and Rabinovici showed that, in the context of their models, the phase diagram could be derived by means of a sequence of transformations: duality, charge conjugation and parity. They were the first to show that, together, these three transformations generated the group $S L(2, Z)$ and that the phases are characterized by condensates with oblique confinement. The phase diagram of the FQHE exhibits, phenomenologically, a very similar structure. Although a microscopic model to motivate such constructions for systems related to the FQHE has not been available, the analysis of the phase diagram by Kivelson, Lee and Zhang [8] and by Ross and Lütken [11] has followed a line very close to that of Cardy and Rabinovici [39].

We now proceed to derive a duality transformation of this theory by standard methods [29,36 38]. This amounts to rewriting the theory in terms of a dual theory defined in terms of loops on the dual lattice. Since the flux loops reside on the links of the dual lattice,

duality, as usual, is the same as to exchange electric currents with magnetic fluxes. By means of the Poisson Summation formula, the partition function can be written in the form

$$
\mathcal{Z}=\sum_{\left\{m_{\mu}\right\}} \int \mathcal{D} \ell_{\mu} \prod_{\vec{r}} \delta\left(\Delta_{\mu} \ell_{\mu}\right) e^{-S\left[\ell_{\mu}\right]+2 \pi i \sum_{\vec{r}} m_{\mu}(\vec{r}) \ell_{\mu}(\vec{r})}
$$

where $m_{\mu}(\vec{r})$ takes values on the integers and $\ell_{\mu}(\vec{r})$ are now real numbers.

We will assume that the system obeys periodic boundary conditions in the time direction $\left(x_{3}\right)$. For simplicity, we will also assume that the system obeys periodic boundary conditions in space (this choice does not affect the physics of this problem in any significant way). The 
configurations of loops can be classified by the number of times they wind around the time direction $\left(x_{3}\right)$. Since we will always work in the thermodynamic limit and will only be interested in systems at zero total center of mass momentum and current, it is not necessary to consider configurations which wind around the space directions. However, if we want to describe systems at non-zero density (i. e. without Charge Conjugation invariance) we need to consider configurations with non-zero winding number around the time direction.

The constraint on the field $\ell_{\mu}(\vec{r})$ can be solved in terms of a topologically trivial piece and a field $\ell_{\mu}^{\text {top }}(\vec{r})$ which accounts for the the loops with non-zero winding number. The topological loops are important to describe systems at non-zero matter density . The constraint is solved by

$$
\ell_{\mu}(\vec{r})=\epsilon_{\mu \nu \sigma} \Delta_{\nu} \phi_{\sigma}(\vec{R})+\ell_{\mu}^{\mathrm{top}}(\vec{r})
$$

In what follows, the topological loops $\ell_{\mu}^{\text {top }}$ will be set to zero. Notice that, in solving the constraint, a gauge invariance has now appeared; since if $\phi_{\sigma}(\vec{R})$ solves the constraint, so does $\phi_{\sigma}(\vec{R})+\Delta_{\sigma} F(\vec{R})$, where $F(\vec{R})$ is an arbitrary function of $\vec{R}$. This gauge symmetry of the dual theory will require the use of a gauge fixing condition when we integrated out the variables $\phi_{\sigma}$.

We now carry out the duality transformation. It is convenient to do it in Fourier space. In terms of the Fourier transforms of $\ell_{\mu}$ and $\phi_{\mu}$ (with wavevector $k_{\mu}$ ), we get

$$
\ell_{\mu}(k)=i \epsilon_{\mu \nu \sigma} \Delta_{\nu}(k) \phi_{\sigma}(k)
$$

where

$$
\Delta_{\nu}(k) \equiv-i\left(e^{i k_{\nu}}-1\right)
$$

is a lattice derivative. From now on we will use the notation $\Delta_{\nu}(k) \equiv k_{\nu}$.

We now specify the kernels $G_{\mu \nu}$ and $K_{\mu \nu}$. Without any loss of generality, the Fourier transforms of the kernels, $G_{\mu \lambda}(k)$ and $K_{\mu \lambda}(k)$ can be taken to be of the form

$$
\begin{aligned}
\tilde{G}_{\mu \lambda}(k) & =g(k) T_{\mu \lambda}(k) \\
\tilde{K}_{\mu \lambda}(k) & =\frac{f(k)}{\sqrt{k^{2}}} C_{\mu \lambda}(k),
\end{aligned}
$$


where $T_{\mu \lambda}(k)$ and $C_{\mu \lambda}(k)$ are the parity-even and parity-odd (Chern-Simons) tensors,

$$
T_{\mu \lambda}(k)=\delta_{\mu \lambda}-\frac{k_{\mu} k_{\lambda}}{k^{2}} \quad C_{\mu \lambda}(k)=i \epsilon_{\mu \lambda \rho} \frac{k_{\rho}}{\sqrt{k^{2}}} .
$$

The coupling functions $g(k)$ and $f(k)$ define the theory. Notice that since $\ell_{\mu}(k)$ is a conserved current, a term in $G_{\mu \nu}$ proportional to the $k_{\mu} k_{\nu}$ does not actually contribute to the action.

We will consider two models, defined by two distinct choices for $g(k)$. In the first case, which we will call Model I, we choose $g(k) \equiv g$. This choice describes systems with short range interactions. In the other case, which we call Model II, we choose $\tilde{G}_{\mu \nu}(k)$ to be long ranged, i. e. $\lim _{k_{\mu} \rightarrow 0} \sqrt{k^{2}} g(k)=g$. As explained in the Introduction, this choice corresponds to the non-local (retarded) $1 /|x|^{2}$ interactions induced by the quantum fluctuations of the electromagnetic field (in the full $3+1$-dimensional space-time) on the particles that move on the plane. We will show below that Model II is actually a self-dual theory. The coupling constants of these theories will be given by the zero momentum limits of the functions.

We now complete the duality transformation. The dual theory is defined in terms of the integer-valued dual field $L_{\mu}(\vec{R})$

$$
L_{\mu}(\vec{R})=\epsilon_{\mu \nu \sigma} \Delta_{\nu} m_{\sigma}(\vec{r})
$$

The dual theory is found by integrating out the field $\phi_{\mu}$. Because this theory is gauge invariant, we must add a gauge fixing term in order to obtain a finite result. The final answer is, naturally, independent of the choice of gauge [40].

Explicitly, we find that the partition function can be written in the form

$$
\mathcal{Z}_{\mathrm{D}}\left[A_{\mu}(\vec{r})\right]=\mathcal{Z}_{0} \sum_{\left\{L_{\mu}\right\}} e^{-S_{\mathrm{D}}\left[L_{\mu}\right]} \prod_{\vec{R}} \delta\left(\Delta_{\mu} L_{\mu}(\vec{R})\right)
$$

$S_{\mathrm{D}}\left[L_{\mu}\right]$ is the dual action and it is given by

$$
\begin{aligned}
S_{\text {dual }}\left[L_{\mu}\right] & =\frac{1}{2} \sum_{\vec{R}, \vec{R}^{\prime}} L_{\mu}(\vec{R})\left(G_{D}^{\mu \nu}\left(\vec{R}-\vec{R}^{\prime}\right)+i K_{D}^{\mu \nu}\left(\vec{R}, \vec{r}^{\prime}\right)\right) L_{\nu}\left(\vec{R}^{\prime}\right) \\
& +i \sum_{\vec{R}, \vec{r}^{\prime}} e_{D}\left(\vec{R}-\vec{r}^{\prime}\right) L_{\mu}(\vec{R}) A_{\mu}\left(\vec{r}^{\prime}\right)+\sum_{\vec{r}, \vec{r}^{\prime}} h_{D}\left(\vec{R}-\vec{R}^{\prime}\right) L_{\mu}(\vec{R}) B_{\mu}\left(\vec{R}^{\prime}\right) \\
& +\frac{1}{2} \sum_{\vec{r}, \vec{r}^{\prime}} A_{\mu}(\vec{r}) \Pi_{\mu \nu}^{D}\left(\vec{r}, \vec{r}^{\prime}\right) A_{\nu}\left(\vec{r}^{\prime}\right)
\end{aligned}
$$


The dual kernels and charges of eq. (3.9) are in general functions of distance and their Fourier transforms are functions of the momentum. We will simplify our notation by dropping, from now on, any explicit reference to the momentum dependence of the dual kernels and charges.

The dual kernels are given by

$$
\begin{aligned}
G_{\mathrm{D}}^{\mu \nu} & =(2 \pi)^{2} g_{\mathrm{D}} T_{\mu \nu} \\
K_{\mathrm{D}}^{\mu \nu} & =-(2 \pi)^{2} \frac{f_{\mathrm{D}}}{\sqrt{k^{2}}} C_{\mu \nu},
\end{aligned}
$$

where the dual coupling functions $g_{\mathrm{D}}$ and $f_{\mathrm{D}}$ are given by

$$
\begin{aligned}
& g_{\mathrm{D}}=(2 \pi)^{2} \frac{g}{f^{2}+k^{2} g^{2}} \\
& f_{\mathrm{D}}=-(2 \pi)^{2} \frac{f}{f^{2}+k^{2} g^{2}} .
\end{aligned}
$$

Eq. (3.11) is the transformation law of the coupling functions under Duality. Similarly, the dual electric $e_{D}$ and magnetic charges $h_{D}$ are

$$
\begin{aligned}
& e_{D}=\frac{1}{2 \pi}\left(e f_{D}+k^{2} h g_{D}\right) \\
& h_{D}=\frac{1}{2 \pi}\left(-e g_{D}+h f_{D}\right) .
\end{aligned}
$$

$\Pi_{\mu \nu}^{D}$ is the polarization tensor of the dual theory in regimes in which the dual loops are suppressed. Thus, it is an effective renormalization of the tensor $\Pi_{\mu \nu}^{0}$. In general, the full polarization tensor includes contributions coming from both the loops and from the background. $\Pi_{\mu \nu}^{D}$ has the form

$$
\Pi_{\mu \nu}^{D}=\Pi_{D}^{(S)} T_{\mu \nu}-i \Pi_{D}^{(A)} C_{\mu \nu}
$$

where $\Pi_{D}^{(S)}$ and $\Pi_{D}^{(A)}$ are the amplitudes for the parity-even and parity-odd pieces of $\Pi_{\mu \nu}^{D}$,

$$
\begin{aligned}
& \Pi_{D}^{(S)}=\Pi_{0}^{(S)}+k^{2}\left(e^{2}-h^{2} k^{2}\right) \frac{g_{D}}{(2 \pi)^{2}}-k^{2} 2 h e \frac{f_{D}}{(2 \pi)^{2}} \\
& \Pi_{D}^{(A)}=\Pi_{0}^{(A)}-\sqrt{k^{2}}\left(e^{2}-h^{2} k^{2}\right) \frac{f_{D}}{(2 \pi)^{2}}-k^{2} 2 e h \frac{g_{D}}{(2 \pi)^{2}} \sqrt{k^{2}}
\end{aligned}
$$

Hence, the polarization tensor acquires additive corrections under a duality transformation. This feature will play a very important role in the analysis of the conductance. 


\section{ELECTROMAGNETIC RESPONSE}

Using the results of the previous section we can, in principle, determine the long-distance properties of the possible fixed points. We will parametrize the fixed points in terms of their response to electromagnetic perturbations, $i$. e. the longitudinal conductivity $\sigma_{x x}$ and the Hall conductivity $\sigma_{x y}$ for metallic phases and the dielectric constant $\varepsilon$ and the Hall conductivity $\sigma_{x y}$ for the insulating states.

As usual, these responses are determined from the current-current correlation functions. However, the physics of this system is determined naturally by the loop-loop correlation functions

$$
\mathcal{D}_{\mu \nu}\left(\vec{r}-\vec{r}^{\prime}\right)=\left\langle\ell_{\mu}(\vec{r}) \ell_{\mu}\left(\vec{r}^{\prime}\right)\right\rangle
$$

While $\mathcal{D}_{\mu \nu}$ is related to the current-current correlation functions, it is not equal to them. The current correlation functions are instead equal to the full polarization tensor for the system $\Pi_{\mu \nu}\left(\vec{r}-\vec{r}^{\prime}\right)$ which is defined by

$$
\Pi_{\mu \nu}\left(\vec{r}-\vec{r}^{\prime}\right)=\left\langle J_{\mu}(\vec{r}) J_{\nu}\left(\vec{r}^{\prime}\right)\right\rangle=-\left.\frac{\delta^{2}}{\delta A_{\mu}(\vec{r}) \delta A_{\nu}\left(\vec{r}^{\prime}\right)} \log \mathcal{Z}\left(\left\{A_{\mu}(\vec{r})\right\}\right)\right|_{A_{\mu}=0}
$$

where $J_{\mu}$ is the electromagnetic current.

Since the current loops $\left\{\ell_{\mu}\right\}$ are conserved, i. e. $\Delta_{\mu} \ell_{\mu}(\vec{r})=0$ at every site $\vec{r}$, we can always write $\mathcal{D}_{\mu \nu}$ in terms of transverse tensors. Thus, in Fourier space we write

$$
\mathcal{D}_{\mu \nu}(k)=\mathcal{D}^{S}(k ; g, f) T_{\mu \nu}(k)-i \mathcal{D}^{A}(k ; g, f) C_{\mu \nu}(k)
$$

Here, $\mathcal{D}^{S}(k ; g, f)$ and $\mathcal{D}^{A}(k ; g, f)$ are the parity-even and parity-odd amplitudes of $\mathcal{D}_{\mu \nu}$, $T_{\mu \nu}(k)$ and $C_{\mu \nu}(k)$ are the tensors defined in eq. (3.6). Once again, it should be kept in mind that the kernels and the couplings have an explicit momentum dependence.

The relation between the loop correlation function $\mathcal{D}_{\mu \nu}(k)$ and the current correlation function $\Pi_{\mu \nu}(k)$ can be determined by differentiating the partition function. If we also separate the $\Pi_{\mu \nu}$ into parity-even and parity-odd pieces, 


$$
\Pi_{\mu \nu}(k)=\Pi^{S}(k ; g, f) T_{\mu \nu}(k)-i \Pi^{A}(k ; g, f) C_{\mu \nu}(k)
$$

we find

$$
\begin{aligned}
& \Pi^{S}(k ; g, f)=\Pi_{0}^{S}(k)-\left(e^{2}-k^{2} h^{2}\right) \mathcal{D}^{S}(k ; g, f)-2 e h \sqrt{k^{2}} \mathcal{D}^{A}(k ; g, f) \\
& \Pi^{A}(k ; g, f)=\Pi_{0}^{A}(k)-\left(e^{2}-k^{2} h^{2}\right) \mathcal{D}^{A}(k ; g, f)+2 e h \sqrt{k^{2}} \mathcal{D}^{S}(k ; g, f) .
\end{aligned}
$$

A Parity transformation is equivalent to the mapping $g \rightarrow g$ and $f \rightarrow-f$. Hence, under Parity, the amplitudes $\mathcal{D}^{S}(k ; g, f)$ and $\mathcal{D}^{A}(k ; g, f)$ transform like

$$
\begin{aligned}
& \mathcal{D}^{S}(k ; g,-f)=\mathcal{D}^{S}(k ; g, f) \\
& \mathcal{D}^{A}(k ; g,-f)=-\mathcal{D}^{A}(k ; g, f) .
\end{aligned}
$$

Thus, the amplitudes $\Pi^{S}(k ; g, f)$ and $\Pi^{A}(k ; g, f)$ transform in exactly the same way.

By using the definition of the conductivities as linear response coefficients, the components of the conductivity tensor $\sigma_{i j}(i, j=1,2)$ can now be determined by the standard relations (in imaginary time $x_{3}$ )

$$
\sigma_{i j}=\lim _{k_{3} \rightarrow 0} \lim _{\vec{k} \rightarrow 0} \frac{1}{k_{3}} \Pi_{i j}(k) .
$$

Hence,

$$
\begin{aligned}
\sigma_{x x} & =\lim _{k_{3} \rightarrow 0} \frac{1}{k_{3}} \Pi^{S}\left(0, k_{3}\right) \\
\sigma_{x y} & =\lim _{k_{3} \rightarrow 0} \frac{1}{\left|k_{3}\right|} \Pi^{A}\left(0, k_{3}\right),
\end{aligned}
$$

where we have set $\sigma_{11}=\sigma_{22} \equiv \sigma_{x x}$ and $\sigma_{12}=-\sigma_{21} \equiv \sigma_{x y}$. We can now relate $\sigma_{x x}$ and $\sigma_{x y}$ to the coupling constants of this system

$$
\begin{aligned}
\sigma_{x x} & =\sigma_{x x}^{0}+\lim _{k_{3} \rightarrow 0} \frac{1}{k_{3}}\left[-\left(e^{2}-k_{3}^{2} h^{2}\right) \mathcal{D}^{S}\left(k_{3} ; g, f\right)-2 e h\left|k_{3}\right| \mathcal{D}^{A}\left(k_{3} ; g, f\right)\right] \\
\sigma_{x y} & =\sigma_{x y}^{0}+\lim _{k_{3} \rightarrow 0} \frac{1}{k_{3}}\left[-\left(e^{2}-k_{3}^{2} h^{2}\right) \mathcal{D}^{A}\left(k_{3} ; g, f\right)+2 e h\left|k_{3}\right| \mathcal{D}^{S}\left(k_{3} ; g, f\right)\right] .
\end{aligned}
$$

If the system is in a metallic phase both conductivities $\sigma_{x x}$ and $\sigma_{x y}$ are in general different from zero. If the system has an energy gap and, hence, it is incompressible, the longitudinal 
conductivity $\sigma_{x x}$ vanishes. Instead, $\Pi^{S}(k) / k^{2}$ has a finite limit as $k \rightarrow 0$ which can be identified with the inverse of the effective dielectric constant, $\varepsilon^{-1} \equiv \lim _{k \rightarrow 0} \Pi^{S}(k) / k^{2}$ of the system in an insulating phase. If the insulator has broken Time Reversal symmetry, as in the FQHE, the Hall conductivity $\sigma_{x y}$ may be non-zero.

The phase diagram of the system is spanned by the asymptotic behavior at small momenta of the coupling functions $g(k)$ and $f(k)$, and of the electric and magnetic charges $e(k)$ and $h(k)$. In the next section we will discuss two distinct limiting behaviors for $g$ which yield quite different physics. In general, the conductivity tensor at momentum scale $k$ is a function of $g, f, e$ and $h$. In the next section we will exploit the existence of these duality transformations to determine the properties of both the stable phases of this system and of the phase transitions which separate the phases.

\section{PHASE DIAGRAM}

In this section we will apply the general duality results of section III to Models I and II and use it to determine the qualitative features of the phase diagram. In this paper we will considering only theories with Charge Conjugation invariance. In this case the total charge $Q$ is set to zero and only loops that close on themselves are taken into account.

\section{A. Short Range Interactions}

Let us consider first the case of Model I. In this model, the interactions are short ranged and the effective action is characterized by finite limiting values of the coupling constants. Hence, we choose the parametrization

$$
\begin{aligned}
& g=\lim _{k \rightarrow 0} \frac{g(k)}{2 \pi} \\
& f=\lim _{k \rightarrow 0} \frac{f(k)}{2 \pi}
\end{aligned}
$$

This choice of parametrization reflects the fact that since the interaction is short ranged, $g(0) \neq 0$. Also, $f(0)$ is the statistical angle and as such it is defined modulo $2 \pi$. Thus, 
Periodicity is the shift of $f$ by integers, $i . e . f \rightarrow f+n$.

Under Duality, the coupling constants of Model I transform like

$$
\begin{aligned}
& g_{\mathrm{D}}=\frac{g}{f^{2}+k^{2} g^{2}} \\
& f_{\mathrm{D}}=-\frac{f}{f^{2}+k^{2} g^{2}}
\end{aligned}
$$

Eq. (5.2) shows that if $f \neq 0$, the effective dual couplings as $k \rightarrow 0$ become

$$
\begin{aligned}
g_{\mathrm{D}} & =\frac{g}{f^{2}} \\
f_{\mathrm{D}} & =-\frac{1}{f}
\end{aligned}
$$

The electric and magnetic charges obey similar transformation laws

$$
\begin{aligned}
& e_{D}=\frac{1}{2 \pi} \lim _{k \rightarrow 0}\left(e f_{D}(k)+k^{2} h g_{D}(k)\right)=e f_{D} \\
& h_{D}=\frac{1}{2 \pi} \lim _{k \rightarrow 0}\left(-e g_{D}(k)+h f_{D}(k)\right)=-e g_{D}+h f_{D}
\end{aligned}
$$

Thus the theory is self-dual in the sense that the original theory and its dual have the same form. Clearly, since by shifts of the stastitical angle it is always possible to bring $f$ to the fundamental period $0<f \leq 1$, the effective dual coupling satisfies the inequality $g_{\mathrm{D}} \geq g$ and the dual coupling constant is larger that the original one. One usually expects that, if the original coupling constant is large, the dual coupling constant should be small; if this property holds, the strong and weak coupling regimes are related by duality. In this case, the analysis of these limits becomes very simple since in one regime the loops are suppressed while in the other the dual loops are suppressed. However, in general, the coupling constants of Model I, defined in eq. (5.2) and (5.3), do not have this property. Hence, if the coupling constant of the original theory is of order one, the dual coupling is generally larger than one. Therfore, for Model I duality does not help (in general) to determine the behavior of the system in asymptotic regimes.

In addition, if $f=0$ (up to integer shifts), the situation is quite different. Now the dual coupling is long ranged $g_{\mathrm{D}}(k)=1 /\left(k^{2} g\right)$. This behavior leads to logarithmic interactions 
of matter loops in real space. The fact that a theory of bosonic loops with short ranged interactions is dual to a theory of loops with Biot-Savart-like logarithmic interactions is well known from the three-dimensional classical $X Y$-model 41]. The loops with long range logarithmic interactions are the vortices of the ordered (or superfluid) phase of the $X Y$ model.

\section{B. Self-Dual Actions}

The transformation properties of Model II under duality can be best studied in terms of the dimensionless coupling constants $f$ (the same as in eq. (5.1)) and $g$ which is given by

$$
f \equiv \lim _{k \rightarrow 0} \frac{f(k)}{2 \pi} \quad g \equiv \lim _{k \rightarrow 0} \frac{\sqrt{k^{2}} g(k)}{2 \pi} .
$$

For space-time separations $x-x^{\prime}$, the interaction kernel $G_{\mu \nu}\left(x-x^{\prime}\right)$ for Model II Model II is transverse and it decays like $g /\left(x-x^{\prime}\right)^{2}$. This is the propagator for Maxwell photons in the full $3+1$-dimensional space-time. Thus, Model II describes a system of charged particles with fractional statistics moving on a plane interacting with the full quantized electromagnetic field (see however, the caveats of reference [28]).

For Model II, the dual coupling constants $f_{\mathrm{D}}$ and $g_{\mathrm{D}}$ are

$$
g_{\mathrm{D}}=\frac{g}{f^{2}+g^{2}} \quad f_{\mathrm{D}}=-\frac{f}{f^{2}+g^{2}}
$$

For the case of Model II, the electric and magnetic charges are required to obey

$$
e \equiv \lim _{k \rightarrow 0} e(k) \quad h \equiv \lim _{k \rightarrow 0} \sqrt{k^{2}} h(k)
$$

The dual electric and magnetic charges $e_{D}$ and $h_{D}$ are

$$
\begin{aligned}
& e_{D}=e f_{D}+h g_{D} \\
& h_{D}=-e g_{D}+h f_{D} .
\end{aligned}
$$

It is convenient to define the complex variable $z \equiv f+i g$. Clearly, while $f$ can take any value (although only rational values are physically meanigful), $g$ has to be a positive real 
number for the interactions to be repulsive and the system to be stable. Hence, the complex variable $z$ takes values on $H$, the upper half of the complex plane.

In terms of $z$, Duality is the mapping

$$
z \rightarrow z_{\mathrm{D}}=-\frac{1}{z}
$$

In this notation, Periodicity becomes the mapping

$$
z \rightarrow z_{\mathrm{D}}=z+n
$$

and Charge Conjugation is now

$$
z \rightarrow z_{\mathrm{D}}=-z^{*}
$$

Notice that it is always possible to use Periodicity to make $\operatorname{Re} z=f \geq 0$. Thus, Charge Conjugation is actually redundant.

Hence, Model II is self-dual in the sense that under Duality it maps into a model with interactions of the same form. Moreover, it has the extended invariance parametrized by the mappings of eq. (5.9), (5.10) and (5.11). These mappings generate the Modular Group $S L(2, Z)$, the group of fractional linear transformations $\mathcal{T}$ with integer coefficients of the upper half of the complex plane $\mathcal{H}$ into itself of the form

$$
\mathcal{T} z=\frac{a z+b}{c z+d}
$$

with $a, b, c, d \in Z$ and $a d-b c=1$. Clearly, the partition function of Model II is Modular Invariant and hence its phase diagram is generated by $S L(2, Z)$. Thus, we expect that Model II should have the conjectured structure of the phase diagram of the FQHE [8,11]. We will show below that Modular Invariance has profound consequences. In particular, it determines the possible behaviors of physical observables such as the conductances.

We also give the transformation rules of the background conductivities under duality transformations. They are

$$
\begin{aligned}
\sigma_{x x}^{D} & =\sigma_{x x}^{0}+\frac{1}{2 \pi}\left[\left(e^{2}-h^{2}\right) g_{D}-2 e h f_{D}\right] \\
\sigma_{x y}^{D} & =\sigma_{x y}^{0}+\frac{1}{2 \pi}\left[-\left(e^{2}-h^{2}\right) f_{D}-2 e h g_{D}\right] .
\end{aligned}
$$


It is convenient to rescale the amplitudes of Eq. (4.9) and to define

$$
d^{S}(g, f)=\frac{2 \pi}{\sqrt{k^{2}}} \mathcal{D}^{S}(g, f) \quad d^{A}(g, f)=\frac{2 \pi}{\sqrt{k^{2}}} \mathcal{D}^{A}(g, f)
$$

The self duality of the theory imposes constraints on rescaled amplitudes $d^{S}(g, f)$ and $d^{A}(g, f)$ and, through them, on the physical conductivities. Thus, from Eq. (4.9), we get that the physical conductivities $\sigma_{x x}$ and $\sigma_{x y}$ at coupling constants $g$ and $f$, for specified electric and magnetic charges $e$ and $h$ are

$$
\begin{aligned}
& \sigma_{x x}=\sigma_{x x}^{0}+\frac{1}{2 \pi}\left[-\left(e^{2}-h^{2}\right) d^{S}(g, f)-2 e h d^{A}(g, f)\right] \\
& \sigma_{x y}=\sigma_{x y}^{0}+\frac{1}{2 \pi}\left[-\left(e^{2}-h^{2}\right) d^{A}(g, f)+2 e h d^{S}(g, f)\right] .
\end{aligned}
$$

Likewise, for a Time Reversal Invariant system, we have the further restriction imposed by Eq. (4.6) on the amplitudes $\mathcal{D}^{S}$ and $\mathcal{D}^{A}$,

$$
\begin{aligned}
& d^{S}(g,-f)=d^{S}(g, f) \\
& d^{A}(g,-f)=-d^{A}(g, f) .
\end{aligned}
$$

We stress here that Time Reversal follows from choosing a specific definition of the loops at short distances in such a way that no fractional spin is assigned to a system with a given fractional statistics. For systems with fractional spin, Time Reversal is broken and the Reflection Symmetry of Eq. (5.16) does not hold.

The transformation properties of the quantities of physical interest become very transparent by using complex notation. Thus we define the complex conductivity $\Sigma$, the amplitude $D$ and the charge $H$,

$$
\begin{aligned}
\Sigma(z) & =\sigma_{x y}(f, g)+i \sigma_{x x}(f, g) \\
D(z) & =d_{A}(f, g)-i d_{s}(f, g) \\
H & =h-i e
\end{aligned}
$$

where, for simplicity, we have used the notation $D(z)=D\left(z, z^{*}\right)$. ( Here the stars denote complex conjugation.) Naturally, this notation does not imply that $D(z)$ is necessarily only 
a function of $z$ and thus that it is analytic. In general, the amplitude $D$ is a separate function of $z$ and $z^{*}$ since, a priori, there is no reason to expect $D$ to be an analytic function of the coupling constants $f$ and $g$. However, as we will see below, there are situations of physical interest where it can be locally analytic. We will also see that analyticity has far reaching consequences. In what follows we will write all functions of $f$ and $g$ as functions of $z$. (The same caveats on analyticity still apply.)

With this notation, the complex conductivity $\Sigma$ becomes

$$
\Sigma(z)=\Sigma_{0}+\frac{H^{2}}{2 \pi} D^{*}(z)
$$

Similarly, we find that the duality transformation for the charges is now simply

$$
H_{D}=H z_{D}^{*}
$$

Since this is a self-dual thery, the conductivity of the theory at $z$ and at $z_{D}$ must be the same. Hence

$$
\Sigma(z)=\Sigma_{D}+\frac{H_{D}^{2}}{2 \pi} D^{*}\left(z_{D}\right)
$$

where

$$
\Sigma_{D}=\Sigma_{0}+\frac{H^{2}}{2 \pi} z_{D}^{*}
$$

The consequence of these relations is the the amplitude $D\left(z, z^{*}\right)$ obeys the following functional equation

$$
z^{2} D(z)+z=D\left(-\frac{1}{z}\right)
$$

which is the transformation law of the amplitude $D(z)$ under duality. In addition, $D(z)$ must periodic which here means

$$
D(z)=D(z+1)
$$

Similarly, the Charge Conjugation symmetry transformation of the amplitude under $(f, g) \rightarrow(-f, g)$, Eq. (5.16), which only holds if the system is invariant under Time Reversal, becomes now the Reflection symmetry, 


$$
D\left(-z^{*}\right)=-D^{*}(z),
$$

which expresses the invariance under Time Reversal. Clearly, $D(z)$ cannot be analytic if the system is Time Reversal Invariant.

In the next subsection we will use Modular Invariance (and Time Reversal when appropriate) to determine constraints on the allowed values of $D(z)$. Once $D(z)$ is known, the conductance $\Sigma(z)$ is determined uniquely by Eq. (5.21) once a choice of the electromagnetic coupling $H=h-i e$ is made. In what follows, we will choose the bare model to have $H=-i e$ (i. e. $h=0)$.

\section{Consequences of Modular Invariance}

Let us now discuss the consequences of Modular Invariance. The Modular Group $S L(2, Z)$ is an infinite, discrete, non-abelian group. It has two generators, which we will take to be the Periodicity $P$ and the Duality $S$. Please notice that in the mathematics literature [42], as well as in String Theory [13], the convention is to call $T$-duality what we call Periodicity and $S$-duality what we call Duality. By the action of $S L(2, Z)$, it is always possible to map an arbitrary point of the upper half-plane $\mathcal{H}$ into the Fundamental Domain $F=\left\{z \in \mathcal{H}|| z|\geq 1,| \operatorname{Re} z \mid \leq \frac{1}{2}\right\}$. Particular cases include the mapping the interior of the unit circle $|z|<1$ to the exterior $|z|>1$, which is the conventional mapping of weak and strong coupling regimes into each other.

The unit circle $\mathcal{C}_{1}=\{z ;|z|=1\}$ plays a special role since it is a self-dual manifold although it is not modular invariant. On the unit circle $f^{2}+g^{2}=1$, duality reduces to the mapping $f_{D}=-f$ and $g_{D}=g$. In particular, the self-dual boson point on the unit circle is $(f, g)=(0,1) \equiv i$ and the self-dual fermion point on the unit circle is $(f, g)=$ $(1 / 2, \sqrt{3} / 2) \equiv \rho$. The boson and fermion self-dual points have very special properties. Let $[i]$ and $[\rho]$ denote the sets of points of the upper half-plane $\mathcal{H}$ which are the images of $i$ and $\rho$ under $S L(2, Z)$ respectively. For similar reasons, we will call the sets of points with integer real parts ( $i$. e. $f \in Z$ ) the boson lines. Likewise, the the sets of points with half-integer 
real parts $i$. e. $\left.f \in Z+\frac{1}{2}\right)$ are the fermion lines. In particular, $\operatorname{Re}[z]=1$ will be the boson line while $\operatorname{Re}[z]=\frac{1}{2}$ is the fermion line.

In addition of the finite modular fixed points $[i]$ and $[\rho]$ the modular group also has fixed points at extreme regimes of the upper half plane. The extreme fixed points are the point $\{i \infty\}$ and $[\infty]$, the set $Q$ of rational points on the real axis. The point $\{i \infty\}$ is the limit $\operatorname{Im} z \rightarrow \infty$ and corresponds to the strong coupling limit $g \rightarrow \infty$. In this regime the loops are suppressed. Conversely, [ $\infty$ ] is the set of points of $\mathcal{H}$ with $g=0$ and $f$ rational. These points map into each other under $S L(2, Z)$ and are also images of the point at infinity on the real axis. Also, since $g=0$, physically the set of points $[\infty]$ corresponds to systems of non-interacting charged particles. Also, since $g \rightarrow 0$, for these points it is not legitimate to neglect other, shorter range, interactions. In fact, close enough to these points, Model II behaves like Model I. Clearly, the behavior near these points is quite simple since, for large values of $g$ the loops are suppressed while for small values of $g$ the dual loops are suppressed.

The bosonic point $i$ and the fermionic point $\rho$ are examples of fixed points of $S L(2, Z)$ and describe interacting systems. It can be shown [44] that the set $\mathcal{C}$ of all points of the upper half-plane $\mathcal{H}$ which are fixed points under the Modular Group $S L(2, Z)$ (namely the set of points in $\mathcal{H}$ which are fixed points of a modular transformation), is the union of $[i]$, $[\rho]$ and $[\infty]$, i. e. $\mathcal{C}=[i] \cup[\rho] \cup[\infty]$. In what follows we will say that a fixed point is bosonic or fermionic if it is in $[i]$ or $[\rho]$ respectively. Notice that there will be points on fermion lines which are bosonic since they are images of $i$, for example $z=\frac{1}{2}+\frac{i}{2}$.

These three families of modular fixed points should determine the qualitative global features of the phase diagram. This fact has been stressed by Ross and Lütken [11], and by Lütken [12] who have given a detailed description of these modular fixed points. In that work, they proposed a set of phenomenological Renormalization Group flows and fixed points which are compatible with the requirement of modular invariance. Thus, Ross and Lütken were led to the conclusion that the RG fixed points should also be $S L(2, Z)$ or modular fixed points. Thus, the set $[\infty]$ describes plateaus while the finite fixed points $[i]$ and $[\rho]$ describe phase transitions. The qualitative phase diagram of Model II has the same 
qualitative structure as the phase diagram of Lütken [12] (see in particular Lütken's figure 2). This is also the phase diagram that has been proposed by KLZ for the Fractional Quantm Hall Effect [8]. However, we will show evidence that suggests that Model II has properties that we would normally assign to critical points at most points of the parameter space $\mathcal{H}$.

Before proceeding further it is convenient write down the transformation law of the complex conductance $D(z)$ for an arbitary modular transformation. The transformation laws for $D(z)$ under duality Eq. (5.22) and periodicity Eq. (5.23), generalize for an arbitrary modular transformation $\mathcal{T} \in S L(2, Z)$ of the form of Eq. (5.12), to the following law

$$
D\left(\frac{a z+b}{c z+d}\right)=(c z+d)^{2} D(z)+c(c z+d)
$$

Once again, we stress that so far we have not made any assumptions on the analytic properties of $D(z)$.

Equation (5.25) has a very striking and suggestive form. Since the partition function is invariant under the action of $S L(2, Z)$, naively we would have expected that the complex amplitude $D(z)$ should also be invariant under $S L(2, Z)$. However, the invariance of the partition function does not require the invariance of correlation functions. Instead, only covariance is actually required. This is precisely what happens in critical phenomana where scale invariance only requires that correlation functions to exhibit scaling behavior. Thus, in principle, we could have anticipated that if $D(z)$ was not a modular invariant, it would instead transform homogeneously under the action of the Modular Group $S L(2, Z)$. Functions $F_{k, \bar{k}}\left(z, z^{*}\right)$, labelled by two integers $k$ and $\bar{k}$, that transform homogeneously under $S L(2, Z)$ as

$$
F_{k, \bar{k}}\left(\mathcal{T} z,(\mathcal{T} z)^{*}\right)=(c z+d)^{k}\left(c^{*} z^{*}+d^{*}\right)^{\bar{k}} F_{k, \bar{k}}\left(z, z^{*}\right)
$$

are said to be modular of weight $(k, \bar{k})$. In the special case when the function $F(z)$ is holomorphic (and $k=\bar{k}$ ), it transforms like

$$
F_{2 k}(\mathcal{T} z)=(c z+d)^{2 k} F_{2 k}(z)
$$


and it is called a modular form of weight $2 k$. Typical examples of modular forms are the Eisenstein series $G_{2 k}(z)$

$$
G_{2 k}(z)=\sum_{n, m \in Z}{ }^{\prime} \frac{1}{(n z+m)^{2 k}}
$$

and their generalizations 44 .

However, Eq. (5.25) transforms inhomogeneously, i. e. in addition to the scale factor of the form of Eq. (5.27) (with weight $2 k=2$ ), there is an extra additive contribution. This feature can be traced back to the duality transformation properties of the polarization tensor. Indeed, as Eq. (3.14) shows, an external electromagnetic perturbation (no matter how weak) breaks self-duality. We will see shortly that $D(z)$ is related to a (loosely defined) modular form of weight 2 . This inhomogeneous transformation law has a striking similarity with the transformation law of the energy-momentum (or stress-energy) tensor in two-dimensional Conformal Field Theory which exhibits a Conformal Anomaly [45]. By analogy we can regard the inhomogeneous transformation law of Eq. (5.25) as a Modular Anomaly.

It is straightforward to show that if $z_{0}$ is a fixed point, the generalized transformation law Eq. (5.25) determines uniquely the value of $D\left(z_{0}\right)$ at the fixed points. Indeed, let $z_{0} \in \mathcal{C}$ and let $\mathcal{T}_{z_{0}}$ be the modular transformation that leaves $z_{0}$ invariant, i. e. $\mathcal{T}_{z_{0}} z_{0}=z_{0}$. Then Eq. (5.25) reads

$$
D\left(\mathcal{T}_{z_{0}} z_{0}\right)=D\left(z_{0}\right)=\left(c z_{0}+d\right)^{2} D\left(z_{0}\right)+c\left(c z_{0}+d\right)
$$

Hence we find

$$
D\left(z_{0}\right)=\frac{c\left(c z_{0}+d\right)}{1-\left(c z_{0}+d\right)^{2}}
$$

The value of $D\left(z_{0}\right)$ of Eq. (5.30) determines uniquely the allowed values of the conductivities at the fixed points. However, it is straightforward to check that

$$
D(z)=\frac{i}{2 \operatorname{Im} z}
$$

satisfies both the requirement of periodicity $P$ and it transforms correctly under duality $S$. Hence, the value of $D(z)$ at the fixed points given by Eq. (5.30) must be equal to 


$$
D\left(z_{0}\right)=\frac{i}{2 \operatorname{Im} z_{0}}
$$

Therfore, $D(z)$ is pure imaginary at the fixed points.

Let us derive its consequences for the specially important cases of bosons and fermions:

a ) Bosons: The boson fixed point $z_{0}=i$ is left invariant by the Duality transformation, which is the $S L(2, Z)$ transformation with $a=d=0$ and $b=-c=1$. Eq. (5.30) yields the value

$$
D(i)=\frac{i}{2}
$$

which is pure imaginary. The conductivities for a theory of self-dual bosons become

$$
\begin{aligned}
& \sigma_{x x}=\sigma_{x x}^{0}-\frac{e^{2}}{2 \pi} d_{S}=\sigma_{x x}^{0}+\frac{e^{2}}{4 \pi} \\
& \sigma_{x y}=\sigma_{x y}^{0}-\frac{e^{2}}{2 \pi} d_{A}=\sigma_{x y}^{0} .
\end{aligned}
$$

Notice that the odd-amplitude $d_{A}=0$. Except for the background conductivities $\sigma_{x x}^{0}$ and $\sigma_{x y}^{0}$, the conductivity $\sigma_{x x}$ of self-dual bosons is indeed universal and equal to $\frac{1}{2} \frac{e^{2}}{2 \pi}$. This is the conjectured value of the universal conductivity.

Likewise, a generic bosonic fixed point (i. e. a fixed point $\left.z_{0} \in[i]\right)$ has the form

$$
z_{0}=\mathcal{T}_{i \rightarrow z_{0}} i \equiv \frac{a i+b}{c i+d} .
$$

Eq. (5.32) tells us that $D\left(z_{0}\right)$ is pure imaginary. The longitudinal conductance at a generic boson fixed point is universal although it is not equal to $\frac{1}{2} \frac{e^{2}}{h}$.

b ) Fermions: The fermion fixed point $z_{0}=\rho=\frac{1}{2}+i \frac{\sqrt{3}}{2}$ is left invariant by Duality followed by Periodicty (with period 1). This is the modular transformation with $a=-b=c=1$ and $d=0$. For this point Eq. (5.30) yields the value

$$
D(\rho)=i \frac{\sqrt{3}}{3}
$$


which, once again, is pure imaginary. The conductivities for self-dual fermions are then given by

$$
\begin{aligned}
& \sigma_{x x}=\sigma_{x x}^{0}-\frac{e^{2}}{2 \pi} d_{S}=\sigma_{x x}^{0}+\frac{e^{2}}{2 \pi} \frac{\sqrt{3}}{3} \\
& \sigma_{x y}=\sigma_{x y}^{0}-\frac{e^{2}}{2 \pi} d_{A}=\sigma_{x y}^{0} .
\end{aligned}
$$

Thus, the conductivities are again universal since, up to effects of the background conductivites, we get $\sigma_{x y}=0$ and $\sigma_{x x}=\frac{e^{2}}{2 \pi} \frac{\sqrt{3}}{3}$ as the value of the critical conductivity for this self-dual fermion system. This analysis can be extended to all the fermionic fixed points, $z_{0} \in[\rho]$. Once again Eq. (5.32) yields a universal conductace.

It is interesting to consider the fixed points (both bosonic and fermionic) on the fermion line $z=\frac{1}{2}+i g$. These points are mapped into other points on the same fermion line $z^{\prime}=\frac{1}{2}+\frac{i}{4 g}$ by the modular transformation $(a, b, c, d)=(-1,1,-2,1)$. This transformation has one fixed point on this fermion line, namely the bosonic point $z=\frac{1}{2}+\frac{i}{2}$. The transformation law of the amplitude $D(z)$ now becomes

$$
D\left(\frac{1}{2}+\frac{i}{4 g}\right)=-4 g^{2} D\left(\frac{1}{2}+i g\right)+4 i g
$$

It is easy to check that $D\left(\frac{1}{2}+\frac{i}{2}\right)=i$. Likewise, $z=\rho$ maps onto $z=\frac{1}{2}+i \frac{\sqrt{3}}{6}$. The transformation law now yields $D\left(\frac{1}{2}+i \frac{\sqrt{3}}{6}\right)=i \sqrt{3}$. Once again, we find a universal conductance.

c ) Extreme Fixed Points: These are the fixed points in the class $[\infty] \cup\{i \infty\}$. It is straightforward to show that $D(z)$ vanishes near $\{i \infty\}$. For instance, for $z=f+i g$ and $g \rightarrow \infty$, the contribution of the loops to the partition function is suppressed. In the absence of loops the loop correlation functions vanish. More specifically, since the model is periodic in the statistical angle $f$, the partition function can be written as a sum over linking numbers or, what is the same, as the Fourier series

$$
Z(f, g)=\sum_{\nu_{L} \in Z} Z_{\nu_{L}}(g) e^{2 \pi i f \nu_{L}}
$$


Here, the integer $\nu_{L}$ is the linking number and $Z_{\nu_{L}}(g)$ is the partition function at fixed linking number. $Z_{\nu_{L}}(g)$ is positive and it is a function of $g$ alone. For large but finite $g$, each term of this sum is of the order of $\exp \left(-g S_{0}\right)$. The the action $S_{0}$ depends on the shape of the loops but it is finite for large loops. Hence, for large $g$, only small loops will contribute to $Z_{\nu_{L}}(g)$. In this regime, this series is convergent and, in fact the partition function at statistics $f$ is bounded from above by the partition function for bosons (which also has an exponential dependence in $g$, at large $g$ ). Intuitively, this argument seemingly also implies that the leading non-zero contributions to the correlation functions are also exponentially small in $g$. If this were so, in this regime $D(z)$ would have an essential singularity in $g$, for large $g$. While this is perfectly correct for a system of bosons, it is also conceivable that for generic statistics $f$ the phase fluctuations could conspire to modify this behavior even at large $g$. In fact this is what should happen if Eq. (5.32) would hold even at large values of $g$ and not just at the fixed points. Without further elaboration we conclude that, in any case, we should expect $D(z)$ to vanish at large $\operatorname{Im} z$, either eponentially fast or with a weaker power law dependence. In all scenarios, the physics of this system near $\{i \infty\}$ is clearly that of an insulator.

On the other hand, near the other extreme fixed points [ $\infty$ ] (on the real axis $g \rightarrow 0$ ), the loops proliferate. However, since near these points the dual loops are suppressed, the behavior of the system in these regimes can be determined by using the transformation law. For instance, Duality maps the boson points $z=i g$ onto the boson $z=-i / g$. For these points, the transformation law reads

$$
D\left(\frac{i}{g}\right)=-g^{2} D(i g)+i g .
$$

Hence, if $g \rightarrow 0$, this requires the divergent behavior $D(i g) \approx i / g$. Similarly, the points on the fermion line $z=\frac{1}{2}+i g$ for small $g$ are mapped by the modular transformation $(-1,1,-2,1)$ onto points on the same line but now with large values of $g$. Finally, since in the extreme limit $g \rightarrow 0$ the long range interactions effectively disappear, 
the behavior of the system is dominated by short range interactions and/or mass terms. In particular, the free Dirac fermion point $g \rightarrow 0$ has a Parity Anomaly whose precise value is determined by the sign of the (infinitesimal) mass $m$ term [46]. In our language, the Parity Anomaly is the finite real limit $D\left(z=\frac{1}{2}+i 0^{+}\right)=\operatorname{sign}(m) \frac{1}{2}$. We will not elaborate on this interesting issue here any further. In any event, even along the fermion line we expect essential singularities for large $g$ (up to effects of the Parity Anomaly). Notice, however that since the modular fixed points accumulate on the real axis, there will always be a lenght scale in which the long range interaction dominate no matter how small $g$ may be.

The analysis we have just presented shows that, at least for the fixed points, $D(z)$ is completely determined and its values at the fixed points are universal and independent of the analytic properties that we may assign to it. Also, and in the same vein as in the discussion of the Introduction, the fact that $\sigma_{x x}$ is neither zero nor infinite in this system (which is not disordered) implies that, at the modular fixed points, the system must necessarily be critical. This argument suggests very strongly that the system must also be scale invariant at these fixed points.

It is natural to ask if these are the only points of the upper-half plane $\mathcal{H}$ where $\sigma_{x x} \neq 0$, or, equivalently, $\operatorname{Im} D(z) \neq 0$. However, now we have to confront the problem that the transformation law of Eq. 5.25 alone does not determine completely $D(z)$ any longer. To make further progress we need to know something about the analytic properties of $D(z)$.

We have already argued above that if a fractional spin is not assigned to the particles, this system has a time reversal invariance which requires the reflection symmetry of the amplitude $D(z)$. In turn, reflection symmetry implies that $D(z)$ cannot be an analytic function of $z$ in the sense that it is a function of both $z$ and its complex conjugate $z^{*}$. It is natural to assume that there could be a spin-statistics connection for which $D(z)$ is analytic. It is also tempting to speculate that $D(z)$ would become analytic precisely for the natural spin-statistics connection (although we have no proof of this). 
Let us assume momentarily that $D(z)$ is analytic ( holomorphic) and derive the consquences that follow from this assumption. It turns out that, on the upper-half plane $\mathcal{H}$, it is impossible to construct a holomorphic function of $D(z)$ which transform homogeneously under $S L(2, Z)$ like a modular form of weight 2 since this would violate the Residue Theorem. However, in the theory of modular forms [42,44 it is proven that instead there exists a unique holomorphic function which, remarkably, transforms precisely as required by our inhomogeneous transformation law of Eq. (5.25). Thus, even though Model II may not require analiticity it is certainly compatible with it. The precise statement of this result is that $D(z)$ is a holomorphic function of $z$ (hence, only a function of $z$ ) of the form

$$
D(z)=-4 \pi i G_{2}(z)
$$

Here $G_{2}(z)$ is the holomorphic function defined by the Fourier series

$$
G_{2}(z)=-\frac{1}{24}+\sum_{n=1}^{\infty} \sigma_{1}(n) e^{2 \pi i n z}
$$

where $\sigma_{1}(n)$ is the sum of all the divisors of the positive integer $n$,

$$
\sigma_{1}(n)=\sum_{r \mid n} r
$$

In terms of the variable $q=\exp (2 \pi i z)$, the first terms of this Fourier expansion are

$$
G_{2}(z)=-\frac{1}{24}+q+3 q^{2}+4 q^{3}+\ldots
$$

Alternatively, $G_{2}(z)$ can be written as a regularized Eiesenstein series of the form

$$
G_{2}(z)=-\frac{1}{8 \pi^{2}} \lim _{\epsilon \rightarrow 0^{+}} \sum_{m, n}{ }^{\prime} \frac{1}{(m z+n)^{2}|m z+n|^{\epsilon}}+\frac{1}{8 \pi \operatorname{Im} z}
$$

The significance of Eq. (5.41) is that, if analyticity were to hold exactly, the complex conductance would be a universal function of $z=f+i g$, completely determined on the entire upper-half plane.

In contrast, if analyticity does not hold, many options are available. Indeed, we have already noticed that 


$$
D(z)=\frac{i}{2 \operatorname{Im} z}
$$

is a solution. It also happens to satisfy reflection symmetry and it is also consistent with all the values of $D(z)$ that we have determined so far, including the asymptotic behavior for $g \rightarrow 0$. However, we now have infinitely many solutions available to us. Indeed, let $F(z)$ be a modular invariant (not necessarily holomorphic) function of $z$. An example of such functions is the Jacobi Invariant $J(z)$ (which has an essential singularity at infinity). The derivative $F^{\prime}(z)$ is modular of weight 2 (but not holomorphic). Then we have

$$
D(z)=\frac{i}{2 \operatorname{Im} z}+F^{\prime}(z) .
$$

For reflection symmetry to hold it is sufficient to write

$$
D(z)=\frac{i}{2 \operatorname{Im} z}+i\left|F^{\prime}(z)\right|,
$$

which satisfies all the requirements.

Thus, analyticity is a very strong (and highly desirable!) constraint which determines uniquely the amplitude $D(z)$ and the conductances for all points of the upper half-plane. Analyticity appears to be related to the existence of a spin-statistics connection in a manner which is not yet fully understood. If analyticity holds, our results show that it is possible to have a more general (and interesting) type of superuniversality: the conductances are a universal function of the coupling constant $g$ and of the statistical angle $f$. In a sense, the complex conductance behaves very much like a universal amplitude in critical phenomena, which are universal functions of effective coupling constants. The latter, of course, are related in a highly non-universal manner to the coupling constants that define the physical systems at microscopic scales. The same structure appears to hold here. We close by stressing that, even if analyticity does not hold, modular invariance alone is sufficient to prove that the complex conductance is different, but determined, at the different modular fixed points. 


\section{PERIODICITY IN QFT AND CRITICAL BEHAVIOR}

In previous sections we derived a number of duality transformations for two classes of theories of interacting loops endowed with statistical phases.

We showed that, for Model I and for a subset of possible values of the statistical angle $f$, the theory with short range interactions among loops is dual to a theory with long range, logarithmic, interactions among loops. The theory with short range interactions among loops is essentially identical to the high temperature phase of the 3D $X Y$ classical Model. In the high temperature phase, the loops are the loops of the diagrams of the high temperature expansion. These diagrams are also a lattice version of the Feynaman diagrams of a theory of a self-interacting complex field. Close to the critical coupling constant (or $T_{c}$ ) this theory becomes equivalent to $|\phi|^{4}$ theory. This picture is known to hold from detailed studies of critical behavior both within the $4-\epsilon$ expansion and directly in three dimensions [55]. The dual theory is the low temperature phase of the 3D $X Y$-Model. In this regime the loops are vortex defects of the order parameter field. The fact that a theory with long range interactions among loops is dual to a theory with short range loop interactions, both separated by the same second order phase transition, indicates that the long range field of the vortices of the low temperature phase is actually screened and that the actual fixed point is that of the $|\phi|^{4}$ theory. The critical behavior of this theory is controlled by the WilsonFisher fixed point of its $4-\epsilon$ expansion. It is well established that the phase transition of the 3D XY model is second order. The disordered phase can be viewed as a vortex condensate but, unlike the 2D $X Y$ model, it is not a vortex-liberation phase transition. In fact, as the transition is approached, core size of the core of the vortices grows large and the long range interaction of the vortices becomes screened. The transition of the 3D $X Y$ model is actually controlled by the Wilson-Fisher fixed point of $|\phi|^{4}$ Landau theory. For other values of the statistical angle $f$, the theory is equivalent to a $|\phi|^{4}$ theory coupled to a Chern-Simons gauge field with a suitable value of the Chern-Simons coupling constant.

If matter is second-quantized, the situation is somewhat less clear. For second-quantized 
theories defined on a lattice 474 49,35, Periodicity always holds and so does Charge Conjugation in the appropriate cases. For non-relativistic dense systems in two-dimensional continuum space ( $i$. e. all Chern-Simons theories of the FQHE) are invariant under $P$ and invariance under $C$ only happens under special circumstances. For continuum relativistic field theories the situation is less clear since the symmetries depend on subtle properties of this theory at short distances and are thus strongly sensitive to regularization prescriptions. For instance, field theories with a single relativistic fermi field with a four-fermi interaction and coupled to a Chern-Simons gauge theory have been considered recently by several authors [50 52]. Within a perturbative approach, which uses dimensional regularization, these authors study the critical behavior of such theories. In particular, it is found [52 that the dimension of the four-fermi operator $\Delta_{4}$ is a continuous function of the statistical angle $\delta$ (related to the Chern-Simons coupling constant $\theta=1 / 2 \delta$ ) and that, in particular, $\Delta_{4}$ decreases like $\Delta_{4}-4 \sim \delta^{2}$ (here 4 is the dimension of the four-fermi operator at the free field fixed point). Moreover, due to the topological charcter of the Chern-Simons action, its coupling constant acquires at most finite renormalizations [53] and, consequently, it does not flow under the renormalization group. Thus, if taken at face value, these results seem to imply that the critical properties of at least relativistic matter fields coupled to ChernSimons gauge fields do depend parametrically on the value of the Chern-Simons coupling constant $\theta$ and that these transitions are neither necessarily superuniversal nor periodic in $\delta$.

The results of references [50 52] hold in a perturbative expansion in powers of $\delta(i . e$. the deviation from fermions). Hence, periodicity cannot possibly be respected even if the full theory had this symmetry. However, the use of dimensional regularization imposes more serious limitations. As a regularization procedure, dimensional regularization yields unambiguous results only for theories (and observables) which can be continued in dimension in a natural way. However, dimensional regularization is notoriously unreliable for the study of theories with operators which involve the Levi-Civita epsilon tensor, such as Chern-Simons gauge theory itself and many others. The dimensional continuation of operators with the 
epsilon tensor is plagued with ambiguities with additional (or evanescent) operators which make important contributions even though they formally do not exist in three dimensions. From a physical point of view, the vanishing of all divergent contributions other than logarithmic, which in standard problems is an advantage of dimensional regularization, turns into a problem in this case. For instance, in these theories there are ultraviolet linearly divergent contributions to the fermion self-energy which induce a non-zero mass for the fermion. Since fermion masses break parity and time reversal in $2+1$-dimensions, they alter the symmetries of the system. Thus, different choices of cutoff may affect the physics. For instance, in reference [54] the anisotropic quantum Heisenberg antiferromagnet was studied using a lattice Chern-Simons approach. This theory is a lattice version of the relativistic theory. It was found [54 that the parity breaking induced fermion mass terms are essential for the spectrum of the theory to agree with the spectrum rigurously known for the anisotropic quantum Heisenberg antiferromagnet.

In any event, a full non-perurbative construction of these continuum field theories in which Periodicity is an explicit symmetry is still missing. Our discussion in the rest of the paper suggests a possible way to do this. Our results indicate that perturbation theory may be misleading.

\section{CONCLUSIONS}

In this paper we constructed of a set of models which qualitatively describe the transitions between quantum Hall plateaus and related problems. We gave an explicit construction of a model (Model II) which is invariant under periodic shifts of the statistical angle and under duality transformations. In fact the model is invariant under the full Modular Group $S L(2, Z)$. We also investigated the transformation laws under $S L(2, Z)$ of physical correlation functions. In particular we showed that the complex conductance transforms (loosely speaking) like a modular form of weight 2. This observation alone enabled us to determine

the values of the complex conductance at the fixed points of the modular group. The values 
of the conductances at these fixed points are universal but not constant. We present an argument that shows that the system is critical at least at these points. In a sense the conductance behaves like a universal amplitude of a critical system. Further assumptions about symmetries have to be made in order to determine the conductance away from the fixed points. If the complex conductance is a holomorphic function of $z=f+i g$, then the modular transformation laws determine the conductance completely on the entire upper half of the complex plane. This is perhaps the strongest form of superuniversality. In fact, all the non-universal physics is encapsulated in the dependence of the (long distance) effective coupling constant $g$ on the microscopic coupling constants. For Time Reversal Invariant systems we find that symmetry arguments alone are no longer sufficient to determine the conductance. It is quite possible that the system should have a hidden supersymmetry for $D(z)$ to be holomorphic.

A number of additional issues remain to be resolved. The models were constructed in such a way that particle-hole symmetry is exact. Also, we have assumed that there was no external magnetic field (on average). Clearly, in order to describe the full set of phase transitions of the FQHE these assumptions need to be removed. In the context of Model I this has already been done in all of the studies of the FQHE plateaus. However, for Model II we have chosen not to consider this generalization here. Although in principle this is straightforward, this generalization already poses an important question. In the context of Model II we have shown that at the modular fixed points that complex conductance is pure imaginary and this implies the vanishing of the Hall conductance in a modular invariant system. This result followed from the fact that the complex conductance transforms like a modular form of weight 2 . We have also observed that, in the presence of an electromagnetic field, the partition function is no longer a modular invariant. At finite fields and chemical potential, the partition function will transform in a non-trivial way. Thus, away from perfect particle-hole symmetry, the system can have a non zero value of $\sigma_{x y}$ at the fixed points. A related issue is the description in this language of the Parity Anomaly in theories of relativistic fermions. 
Finally, there is the question of the unitarity of Model II. Since Model I describes a theory of particles with fractional statistics with short range interactions it can also be written a local field theory of a complex scalar field coupled to a Chern-Simons gauge field. Although the phase transitions of these theories are not understood, their behavior at their stable, semiclassical regimes is well known. In particular, for rational values of the statistical angle, these theories are unitary. In contrast Model II, which turned out to be a more interesting and richer theory, cannot be mapped onto a local field theory for generic values of the statistical angle. Nevertheless, on any bosonic line, the partition function has positive weights and it is unitary. However, on any fermionic line $f=\frac{1}{2}+n$ (with $n \in Z$ ) Model II is also unitary since, on such lines the theory can be related to a non-local version of a Maxwell-Chern-Simons theory. Since Model II is modular invariant, it follows that unitarity should also hold on the images of all of the bosonic and fermionic lines under the full action of $S L(2, Z)$. Therefore, we expect unitarity to hold on a dense set of points of the upper-half plane. However, it is quite possible (and likely) that unitarity may not hold for irrational values of the statistics.

\section{ACKNOWLEDGEMENTS}

We thank L. Chayes, S. Chaudhuri, D. E. Freed, D. H. Lee, C. Lütken, D. Rokhsar and M. Stone for useful discussions. We are also very grateful to L. Pryadko and S. C. Zhang for making their unpublished work available to us. This work was supported in part by the

National Science Foundation through the grants NSF DMR94-24511 at the University of Illinois at Urbana-Champaign and NSF DMR-93-12606 at UCLA. 


\section{REFERENCES}

[1] Xiao-Gang Wen and Qian Niu, Phys. Rev. B41, 9377 (1990).

[2] R. B. Laughlin, Phys. Rev. Lett. 50, 1395 (1983).

[3] F. D. M. Haldane, Phys. Rev. Lett. 51, 605 (1983).

[4] B. I. Halperin, Phys. Rev. Lett. 52, 1583 (1984).

[5] J. M. Leinaas and J. Myrheim, Il Nuovo Cimento 37, 1 (1977).

[6] Xiao-Gang Wen, Phys. Rev. B41, 12838 (1990).

[7] S. C. Zhang, T. H. Hansson and S. Kivelson, Phys. Rev. Lett. 62, 82 (1989).

[8] S. Kivelson, D. H. Lee and S. C. Zhang, Phys. Rev. B46, 223 (1992).

[9] B. Blok and Xiao-Gang Wen, Phys. Rev. B42, 8133 (1990).

[10] J. Frohlich and T. Kerler, Nucl. Phys. B354, 369 (1991); J. Frohlich and A. Zee, Nucl. Phys. B364, 517 (1991).

[11] C. A. Lütken and G. G. Ross, Phys. Rev. B48, 2500 (1993); Phys. Rev. B48, 11837 (1993).

[12] C. A. Lütken, Nucl. Phys. B396, 670 (1993).

[13] E. Manousakis, FSU preprint (1995).

[14] D. Shahar, D. C. Tsui, M. Shayegan, E. Shimshoni and S. Sondhi, Princeton University Preprint (1995) (condmat/9510103).

[15] N. Read, Phys. Rev. Lett. 62, 86 (1989).

[16] X. G. Wen and A. Zee, Phys. Rev. B46, 2290 (1992).

[17] F. Wilczek, Phys. Rev. Lett. 48, 1144 (1982).

[18] Y. S. Wu and A. Zee, Phys. Lett. 147B, 325 (1984). 
[19] E. Witten, Comm. Math. Phys.121, 351 (1989).

[20] A. M. Polyakov, Mod. Phys. Lett. A3 325 (1988).

[21] For a pedagogical discussion see E. Fradkin, Field Theories of Condensed Matter Systems, Addison Wesley, Redwood City (1991).

[22] A. Lopez and E. Fradkin, Phys. Rev. B44, 5246 (1991).

[23] B. I. Halperin, P. A. Lee and N. Read, Phys. Rev. B47, 7312 (1993).

[24] J. Jain, Phys. Rev. Lett. 63, 199 (1989).

[25] It is well known that, for a suitable choice of regularization, the Chern-Simons theory also describes fractional spin [20]. However, this property does depend on the microscopic definition of the theory and it is not universal. For the rest of this paper, the effects of such a fractional spin will be ignored.

[26] L. Pryadko and S. C. Zhang, unpublished, Stanford University Preprint (1995).

[27] A. P. Balachandran, L. Chandar and B. Sathiapalan, unpublished, Syracuse University Preprint (1995).

[28] In a physically relevant model which can describe a condensed matter system, the velocity of the particles is much smaller than the speed of light. A consequence of this is both an anisotropy of the effective interaction and a significant suppression of the coupling constant for the magnetic terms. In this regime, short range interactions (in addition to the instantanoeus Coulomb piece) become dominant. However, below we show that the behavior is highly non-analytic at small coupling. Thus, even there, the magnetic terms are still important even if they are small.

[29] H. A. Kramers and G. H. Wannier, Phys. Rev. 60, 252 (1941); for a review on duality see R. Savit, Rev. Mod. Phys. 52, 453 (1980).

[30] C. J. Callan and D.S. Freed, Nucl. Phys. B 374, 543 (1992); C. J. Callan, A. G. Felce 
and D.S. Freed, Nucl. Phys. B392, 551 (1993).

[31] A. Shapere and F. Wilczek, Nucl. Phys. B320, 669 (1989).

[32] S. J. Rey and A. Zee, Nucl. Phys. B352, 897 (1991).

[33] G. t'Hooft, Nucl. Phys. B190[FS3], 455 (1981).

[34] S. Girvin and A. MacDonald, Phys. Rev. Lett. 58, 1252 (1987).

[35] M. C. Diamantini, P. Sodano and C. Trugenberger, University of Geneva Preprint (December 1994).

[36] M. Peskin, Ann. Phys. (N. Y. ) 113, 122 (1978).

[37] M. Stone and P. Thomas, Nucl. Phys. B144, 513 (1978).

[38] M. P. A. Fisher and D. H. Lee, Phys. Rev. B39, 2756 (1989); D. H. Lee and M. P. A. Fisher, Phys. Rev. B46, 2290 (1992).

[39] J. L. Cardy and E. Rabinovici, Nucl. Phys. B205[FS5], 1 (1982); J. L. Cardy, Nucl. Phys. B205[FS5], 17 (1982).

[40] For simplicity, we fix the gauge by the standard method of adding a gauge fixing term to the action of the form $\frac{\alpha}{2} \sum_{\vec{R}}\left[\Delta_{\mu} \phi_{\mu}(\vec{R})\right]^{2}$. The final expressions, themselves being gauge invariant, are independent of $\alpha$.

[41] T. Banks, R. Myerson and J. Kogut, Nucl. Phys. B129, 493 (1977)

[42] See, for instance D. Zagier, Introduction to Modular Forms, in From Number Theory to Physics, Chapter 4, M. Waldschmidt, J. M. Luck and P. Moussa Editors, SpringerVerlag, Berlin-New York (1992).

[43] For a review see J. Polchinski, What is String Theory, Les Houches Summer School (1994), to be published.

[44] R. A. Rankin, Modular Forms and Functions, Cambridge University Press (1977). 
[45] A. A. Belavin, A. M. Polyakov and A. B. Zamolodchikov, Nucl. Phys. B241, 333 (1984).

[46] S. Deser, R. Jackiw and S.Templeton, Phys. Rev. Lett. 48, 975 (1982); Ann. Phys. 140, $372(1982)$.

[47] J. Frohlich and P. Marchetti, Lett. Math. Phys. 16, 347 (1988).

[48] E. Fradkin, Phys. Rev. Lett. 63, 322 (1989).

[49] D. Eliezer and G. W. Semenoff, Phys. Lett. 286B, 118 (1992).

[50] X. G. Wen and Y. S. Wu, Phys. Rev. Lett. 70, 1501 (1993).

[51] W. Chen, M. P. A. Fisher and Y. S. Wu, Phys. Rev. B48, 13749 (1993).

[52] W. Chen and M. Li, Phys. Rev. Lett. 70, 884 (1993).

[53] G. W. Semenoff, P. Sodano and Y. S. Wu, Phys. Rev. Lett. 62, 715 (1989).

[54] A. Lopez, A. G. Rojo and E. Fradkin, Phys. Rev. B49, 15139 (1994).

[55] A very comprehensive exposition of these results is found in J. Zinn-Justin, Quantum Field Theory and Critical Phenomena, second edition, Oxford University Press (Oxford, UK, 1993) 